\title{
The Demonstration of a Robotic External Leak Locator on the International Space Station
}

\author{
Adam Naids ${ }^{l}$, Dino Rossetti ${ }^{2}$, Tim Bond ${ }^{1}$, Brien Johnson ${ }^{1}$, Alvin Huang ${ }^{3}$, Alexandra Deal ${ }^{3}$, Katie Fox ${ }^{3}$, \\ Michael Heiser ${ }^{3}$, William Hartman ${ }^{3}$, Ronald Mikatarian ${ }^{3}$ \\ ${ }^{1}$ NASA Johnson Space Center, ${ }^{2}$ Conceptual Analytics, ${ }^{3}$ Boeing Space Environments
}

\section{$\underline{\text { Introduction }}$}

The International Space Station (ISS) and all currently conceivable future manned spacecraft are susceptible to mission impacts due to fluid/gas leaks to the exterior environment. For example, there is a well-known risk of ammonia leaks from the ISS External Thermal Control System loops and currently no method to locate them. It was, therefore, critical to develop a method for detecting and locating leaks to preserve vehicle health. The Robotic External Leak Locator (RELL) was developed and deployed to the ISS to provide this capability. An on-orbit validation and demonstration was successfully completed in December 2016 and leak locating operations occurred in February 2017. This paper discusses the results of those exercises including measurements of the environment around ISS, detection of the small ammonia leak and implementation of leak locating methodologies.

RELL is a collaboration between the Goddard Space Flight Center (GSFC) and the Johnson Space Center (JSC) and was launched to the ISS as a Technology Demonstration Payload in December 2015 on Orbital-ATK Commercial Resupply Flight 4.

\section{$\underline{\text { Technology Overview }}$}

RELL consists of two sensors: a Residual Gas Analyzer (RGA) and a Cold Cathode Ion Gauge. The RGA, a small mass spectrometer, provides high sensitivity (1E-12 to 1E-5 Torr). The RGA also provides the ability to identify individual gases which is critical to distinguish the target gas (in this case, ammonia) from gases found in the natural and induced environments. A large spacecraft such as the ISS has a large number of intentional gas vents which contribute to the induced environment and can mask the presence of the leaking gas without the presence of an RGA. The Ion Gauge measures total pressure, has a higher operating pressure range than the RGA (1E-9 to 1 Torr), provides real time pressure measurements and can be used during proximity operations even with larger magnitude leaks. RELL is currently stowed inside the ISS, though an external stowage bay is being considered. To manage costs, the RELL design incorporates COTS hardware that was adapted and ruggedized for application on ISS as shown in Figure 1.

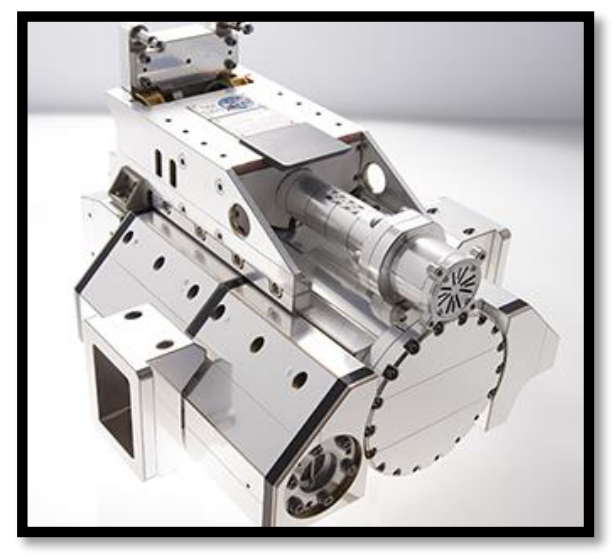

Figure 1: Flight-ready RELL.

The Japanese Experiment Module (JEM) Airlock is used to transfer RELL to the external environment where it is picked up by the Space Station Remote Manipulator System (SSRMS) and Special Purpose Dexterous Manipulator (SPDM) as shown in Figure 2. After SPDM grapples RELL, operations are controlled 
entirely from the ground, minimizing impact to the ISS on-orbit crew. Ground operators maneuver the robotic assets around the ISS in predetermined trajectories based upon potential leak locations. RELL leak locating activities serve as an intermediate step between identification of a suspected leak and leak repair which saves valuable crew Extravehicular Activity (EVA) time for actual repair tasks.

\section{On-Orbit Demonstration}

Originally flown as a Technology Demonstration Payload, the culmination of the project was an on-orbit demonstration that took place in late 2016. This demonstration had a number of goals:

- to assess the sensitivity of the Ion Gauge and RGA combination for locating a leak in the complex ISS environment, including performance of the sensors in space,

- to characterize this environment including both natural gases and gases from ISS,

- to evaluate leak locating methods using the robot capabilities and RELL, and

- to determine factors that would impact the ability to locate leaks on the ISS.

This demonstration occurred November 30 to December 7, 2016 and all objectives were successfully completed. Moreover, an ammonia leak was detected during the demonstration leading to an additional day of operations to identify the leak location. RELL operational data narrowed the suspected leak location areas to the ISS port-side Radiator Beam Valve Module (RBVM) P1-3-2. The instrument performed nominally throughout both the demonstration and leak location operations and the Ion Gauge and RGA proved to be highly sensitive. The data corroborated our understanding of the natural environment and provided measurements of gases vented from the ISS. Lessons learned from the demonstration will be applied to future leak locating activities. This paper and supplemental poster or presentation will discuss the results and findings including some aspects that are critical to leak locating such as diurnal variability in the ammonia signal.

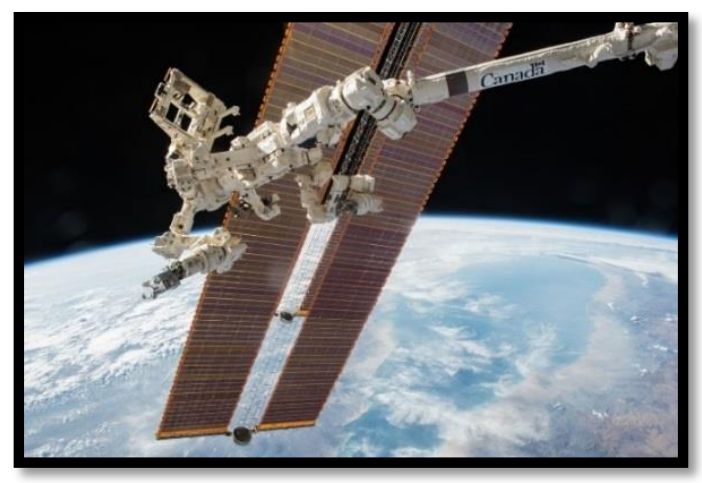

Figure 2: RELL attached to the SPDM during the Demonstration.

\section{Leak Locating Success}

In February 2017, RELL was called back into operation due to a possible increase in the leak rate associated with the $\mathrm{P} 1$ radiator system. The goal was to refine the location of the leak as much as possible. The RELL project team and the ISS robotics community developed and successfully implemented leak locating trajectories covering potential leak sources. During these operations, total pressure data from the Ion Gauge showed increased pressure in a distinct area near a quick disconnect fitting on the aforementioned RBVM P1-3-2. RGA data confirmed ammonia as the driver of the increased pressure. This paper and supplemental poster or presentation will present data from this operation and discuss the process and methodologies employed. Findings from these operations led to an intensive crew inspection during an EVA in March 2017. 